\title{
Seismic Excitation by Single Force and Asymmetric Moment Tensor
}

\author{
Eiji Mochizuki \\ Department of Earth and Planetary Physics, Faculty of Science, \\ The University of Tokyo, Bunkyo-ku, Tokyo 113, Japan
}

\begin{abstract}
Seismic sources are described by the 'traction tensors' which are expressed as the surface integral of traction around the sources.
\end{abstract}

\section{Introduction}

Backus and Mulcahy (1976) have shown that indigenous seismic sources conserving momentum and angular momentum are described by the symmetric second-order tensor, stress glut or moment tensor. However non-indigenous sources have been discussed. Kanamori and his co-workers have studied the single-force excitation which violates the conservation of momentum (e.g., Kanamori and Given, 1982; Kawakatsu, 1989). Recently Takei (1991) has pointed out the possibility of an asymmetric moment tensor which violates the conservation of angular momentum. In this paper, we discuss the mathematical aspects of seismic excitation by non-indigenous sources.

\section{Body-Wave Excitation}

The displacement $u_{i}$ (in the frequency domain) excited by the point force $f_{j}$ is expressed in terms of the Green tensor

$$
u_{i}=G_{i j} f_{j},
$$

where the summation convention is assumed for repeated indices. The Green tensor for a homogeneous and isotropic medium is well-known (e.g., Aki and Richards, 1980). We rewrite the Green tensor in terms of spherical Hankel functions $h_{l}^{(1)}$ (e.g., Abramowitz and Stegun, 1965)

$$
G_{i j}=(i / 4 \pi \rho r)\left\{\alpha^{-2}\left[a_{i j} x h_{0}^{(1)}(x)-c_{i j} h_{1}^{(1)}(x)\right]-\beta^{-2}\left[b_{i j} y h_{0}^{(1)}(y)-c_{i j} h_{1}^{(1)}(y)\right]\right\}
$$

with

$$
a_{i j}=n_{i} n_{j}, \quad b_{i j}=n_{i} n_{j}-\delta_{i j}, \quad c_{i j}=3 n_{i} n_{j}-\delta_{i j}
$$

and

$$
x=\omega r / \alpha, \quad y=\omega r / \beta
$$

Received June 29, 1994; Accepted January 9, 1995 
Here $\omega$ is the frequency, $r$ is the distance from the source, $\rho$ is the density, $\alpha$ is the $\mathrm{P}$-wave velocity, $\beta$ is the S-wave velocity, $n_{i}$ is the direction cosine, and $\delta_{i j}$ is the Kronecker delta.

The excitation due to the moment tensor $M_{j k}$ is given in terms of a spatial derivative for the Green tensor

$$
\begin{aligned}
u_{i}= & -G_{i j, k} M_{j k} \\
= & (i \omega / 4 \pi \rho r)\left\{\alpha^{-3}\left[A_{i j k} x h_{1}^{(1)}(x)-C_{i j k} h_{2}^{(1)}(x)\right]\right. \\
& \left.-\beta^{-3}\left[B_{i j k} y h_{1}^{(1)}(y)-C_{i j k} h_{2}^{(1)}(y)\right]\right\} M_{j k}
\end{aligned}
$$

with

$$
\begin{aligned}
& A_{i j k}=n_{i} n_{j} n_{k} \\
& B_{i j k}=n_{i} n_{j} n_{k}-n_{k} \delta_{i j} \\
& C_{i j k}=5 n_{i} n_{j} n_{k}-n_{i} \delta_{j k}-n_{j} \delta_{i k}-n_{k} \delta_{i j} .
\end{aligned}
$$

We decompose the moment tensor into the symmetric and anti-symmetric parts

$$
M_{j k}=M_{j k}^{(s)}+M_{j k}^{(a)}
$$

with

$$
M_{j k}^{(s)}=\frac{1}{2}\left(M_{j k}+M_{k j}\right), \quad M_{j k}^{(a)}=\frac{1}{2}\left(M_{j k}-M_{k j}\right) .
$$

Two coefficients of Eq. (6) satisfy

$$
A_{i j k}=A_{i k j}, \quad C_{i j k}=C_{i k j} .
$$

We define the symmetric and anti-symmetric parts of $B_{i j k}$.

$$
\begin{aligned}
B_{i j k}^{(s)} & =\frac{1}{2}\left(B_{i j k}+B_{i k j}\right) \\
& =n_{i} n_{j} n_{k}-\frac{1}{2}\left(n_{k} \delta_{i j}+n_{j} \delta_{i k}\right) \\
B_{i j k}^{(a)} & =\frac{1}{2}\left(B_{i j k}-B_{i k j}\right) \\
& =-\frac{1}{2}\left(n_{k} \delta_{i j}-n_{j} \delta_{i k}\right) .
\end{aligned}
$$

Using Eqs.(8) and (10), we obtain

$$
B_{i j k} M_{j k}=B_{i j k}^{(s)} M_{j k}^{(s)}+B_{i j k}^{(a)} M_{j k}^{(a)} .
$$

The excitation due to the anti-symmetric part is restricted to the S-wave expressed in terms of $B_{i j k}^{(a)}$. 


\section{Traction Tensors}

We define the 'traction tensors' which are expressed as the integral of traction $t_{i}$ and coordinate $x_{j}=r n_{j}$

$$
T_{i}=-\int t_{i} \mathrm{~d} S, \quad T_{i j}=-\int t_{i} x_{j} \mathrm{~d} S
$$

where, using the Lame parameters,

$$
t_{i}=\lambda u_{k, k} n_{i}+\mu\left(u_{i, j}+u_{j, i}\right) n_{j} .
$$

The surface integral of Eq. (12) is taken on a small sphere around the source. The angular integral is calculated by formulae

$$
\begin{aligned}
& \left\langle n_{i}\right\rangle=0 \\
& \left\langle n_{i} n_{j}\right\rangle=\frac{1}{3} \delta_{i j} \\
& \left\langle n_{i} n_{j} n_{k}\right\rangle=0 \\
& \left\langle n_{i} n_{j} n_{k} n_{l}\right\rangle=\frac{1}{15}\left(\delta_{i j} \delta_{k l}+\delta_{i k} \delta_{j l}+\delta_{i l} \delta_{j k}\right),
\end{aligned}
$$

where $\langle$.$\rangle denotes the angular average.$

In the vicinity of the source $(r \sim 0)$, the Green tensor and its derivatives are approximated by

$$
\begin{aligned}
G_{i j} \sim & -(8 \pi \rho r)^{-1}\left[\alpha^{-2}\left(n_{i} n_{j}-\delta_{i j}\right)-\beta^{-2}\left(n_{i} n_{j}+\delta_{i j}\right)\right] \\
G_{i j, k} \sim & \left(8 \pi \rho r^{2}\right)^{-1}\left[\alpha^{-2}\left(3 n_{i} n_{j} n_{k}-n_{i} \delta_{j k}-n_{j} \delta_{i k}-n_{k} \delta_{i j}\right)\right. \\
& \left.-\beta^{-2}\left(3 n_{i} n_{j} n_{k}-n_{i} \delta_{j k}-n_{j} \delta_{i k}+n_{k} \delta_{i j}\right)\right] \\
G_{i j, k l} \sim & -\left(8 \pi \rho r^{3}\right)^{-1}\left\{\alpha ^ { - 2 } \left[15 n_{i} n_{j} n_{k} n_{l}-3\left(n_{i} n_{j} \delta_{k l}+n_{i} n_{k} \delta_{j l}+n_{i} n_{l} \delta_{j k}\right.\right.\right. \\
& \left.\left.+n_{j} n_{k} \delta_{i l}+n_{j} n_{l} \delta_{i k}+n_{k} n_{l} \delta_{i j}\right)+\delta_{i j} \delta_{k l}+\delta_{i k} \delta_{j l}+\delta_{i l} \delta_{j k}\right] \\
& -\beta^{-2}\left[15 n_{i} n_{j} n_{k} n_{l}-3\left(n_{i} n_{j} \delta_{k l}+n_{i} n_{k} \delta_{j l}+n_{i} n_{l} \delta_{j k}\right.\right. \\
& \left.\left.\left.+n_{j} n_{k} \delta_{i l}+n_{j} n_{l} \delta_{i k}-n_{k} n_{l} \delta_{i j}\right)-\delta_{i j} \delta_{k l}+\delta_{i k} \delta_{j l}+\delta_{i l} \delta_{j k}\right]\right\} .
\end{aligned}
$$

We first calculate the traction tensors of Eq. (12) for the single force. We use Eqs. (1) and (13)-(15) to obtain

$$
T_{i}=f_{i}, \quad T_{i j}=0 .
$$

The single force is related to the zeroth traction tensor.

For the moment tensor, we use Eq. (5) to obtain

$$
\begin{aligned}
& T_{i}=0 \\
& T_{i j}=\frac{1}{15}(\lambda+2 \mu)^{-1}\left[-(3 \lambda-2 \mu) M_{k k} \delta_{i j}+(12 \lambda+22 \mu) M_{i j}-(3 \lambda+8 \mu) M_{j i}\right] .
\end{aligned}
$$

Vol. 43, No. 1, 1995 
The moment tensor is related to the first traction tensor in a complicated way. We use Eq. (8) to simplify the second of Eq. (17)

$$
\begin{aligned}
& T_{i j}=\frac{4}{3} \mu(\lambda+2 \mu)^{-1} M_{i i} \\
& T_{i j}^{(a)}=M_{i j}^{(a)} \\
& \tilde{T}_{i j}^{(s)}=\frac{1}{15}(9 \lambda+14 \mu)(\lambda+2 \mu)^{-1} \tilde{M}_{i j}^{(s)},
\end{aligned}
$$

where the tilde denotes the deviatoric part.

In the first and third of Eq. (18), additional factors are multiplied for the symmetric moment tensor, the indigenous source of Backus and Mulcahy (1976). On the other hand, Eq. (16) and the second of Eq. (18) are simple for the single force and anti-symmetric moment tensor (see Appendix A). The traction tensors are convenient for the kinematic description of non-indigenous sources, as shown in the next section.

\section{Kinematic Description}

We consider the source region of a small sphere (with radius $a$ ) in which the linear elasticity cannot be applied. In our kinematic description, the traction is assumed to be specified at the surface of the sphere, and the linear elasticity is applied for the exterior of the sphere.

We use the notation $\tau_{i}$ for traction in spherical coordinates $(r, \theta, \phi)$. Following Phinney and Burridge (1973), the canonical components are defined by

$$
\begin{aligned}
& \tau^{0}=\tau_{r} \\
& \tau^{ \pm}=2^{-1 / 2}\left(\mp \tau_{\theta}+i \tau_{\phi}\right) .
\end{aligned}
$$

The canonical components are expanded in terms of the generalized spherical harmonics of Phinney and Burridge (1973)

$$
\tau^{\alpha}(a, \theta, \phi)=\sum_{l m} \tau_{l}^{\alpha m}(a) Y_{l}^{\alpha m}(\theta, \phi) .
$$

We may obtain the displacement outside the source radius on which the traction is given in Eq. (20) as a boundary condition. However, we use an alternative approach in the following.

We also define the canonical components for cartesian coordinates (Mochizuki, 1988)

$$
\begin{aligned}
& t^{0}=t_{3} \\
& t^{ \pm}=2^{-1 / 2}\left(\mp t_{1}+i t_{2}\right) .
\end{aligned}
$$

The relation between cartesian and spherical components is given by

$$
t^{\alpha}=\sum_{\beta} Y_{1}^{\beta \alpha *} \tau^{\beta},
$$


where the asterisk denotes complex conjugation. Similarly, the cartesian coordinates at the surface of the sphere are given by

$$
x^{\alpha}=a Y_{1}^{0 \alpha *} .
$$

We first calculate the zeroth traction tensor

$$
T^{\alpha}=-\int t^{\alpha} \mathrm{d} S=-a^{2} \int t^{\alpha} \mathrm{d} \Omega,
$$

where $\mathrm{d} \Omega=\sin \theta \mathrm{d} \theta \mathrm{d} \phi$. Substituting Eqs. (20) and (22) into Eq. (24), we obtain

$$
T^{\alpha}=-\frac{4}{3} \pi a^{2}\left(\tau_{1}^{0 \alpha}+\tau_{1}^{+\alpha}+\tau_{1}^{-\alpha}\right)
$$

where we use the orthogonality of generalized spherical harmonics. Referring to Eqs. (16) and (25), the single force is related to one of the spheroidal combinations with $l=1$

$$
S_{1}^{(1) m}=\tau_{1}^{0 m}+\left(\tau_{1}^{+m}+\tau_{1}^{-m}\right) .
$$

We next calculate the first traction tensor

$$
T^{\alpha \beta}=-a^{2} \int t^{\alpha} x^{\beta} \mathrm{d} \Omega .
$$

Substituting Eqs. (20), (22), and (23) into Eq. (27), we obtain

$$
T^{\alpha \beta}=-4 \pi a^{3} \sum_{l} \sum_{\gamma}(-1)^{\alpha+\beta+\gamma} \tau_{l}^{\gamma(\alpha+\beta)} \times\left\langle\begin{array}{ccc}
l & 1 & 1 \\
-\gamma & \gamma & 0
\end{array}\right\rangle\left\langle\begin{array}{ccc}
l & 1 & 1 \\
-(\alpha+\beta) & \alpha & \beta
\end{array}\right\rangle,
$$

where the integral for the triple product of generalized spherical harmonics is expressed in terms of Wigner 3-j symbols (e.g., Edmonds, 1960).

Due to the selection rules of $3-j$ symbols, terms with $l=0,1$, and 2 are relevant. Also, we can verify

$$
\begin{aligned}
& T^{\alpha \beta}=T^{\beta \alpha} \quad(l=0 \text { and } 2) \\
& T^{\alpha \beta}=-T^{\beta \alpha} \quad(l=1) .
\end{aligned}
$$

For $l=0$, Eq. (28) reduces to

$$
\begin{aligned}
& T^{00}=-T^{+-}=-\frac{4}{3} \pi a^{3} \tau_{0}^{00} \\
& T^{ \pm 0}=T^{ \pm \pm}=0 .
\end{aligned}
$$

The trace and deviatoric part are given by

$$
\begin{aligned}
& T^{00}-T^{+-}-T^{-+}=-4 \pi a^{3} \tau_{0}^{00} \\
& \tilde{T}^{\alpha \beta}=0 .
\end{aligned}
$$

Referring to the first of Eq. (18), the trace of moment tensor is related to the spheroidal part of traction with $l=0$.

For $l=1$, we obtain an anti-symmetric tensor with 


$$
\begin{aligned}
& T^{+-}=-\frac{2}{3} \pi a^{3}\left(\tau_{1}^{+0}-\tau_{1}^{-0}\right) \\
& T^{ \pm 0}=\mp \frac{2}{3} \pi a^{3}\left[\tau_{1}^{+( \pm 1)}-\tau_{1}^{-( \pm 1)}\right]
\end{aligned}
$$

Referring to the second of Eq. (18), the anti-symmetric part of moment tensor is related to

$$
T_{1}^{m}=\tau_{1}^{+m}-\tau_{1}^{-m}
$$

which is toroidal with $l=1$.

For $l=2$, we obtain a symmetric tensor with

$$
\begin{aligned}
& T^{00}=-\frac{8}{15} \pi a^{3}\left[\tau_{2}^{00}+\frac{1}{2} 3^{1 / 2}\left(\tau_{2}^{+0}+\tau_{2}^{-0}\right)\right] \\
& T^{+-}=\frac{1}{2} T^{00} \\
& T^{ \pm 0}=-\frac{4}{15} 3^{1 / 2} \pi a^{3}\left\{\tau_{2}^{0( \pm 1)}+\frac{1}{2} 3^{1 / 2}\left[\tau_{2}^{+( \pm 1)}+\tau_{2}^{-( \pm 1)}\right]\right\} \\
& T^{ \pm \pm}=-\frac{4}{15} 6^{1 / 2} \pi a^{3}\left\{\tau_{2}^{0( \pm 2)}+\frac{1}{2} 3^{1 / 2}\left[\tau_{2}^{+( \pm 2)}+\tau_{2}^{-( \pm 2)}\right]\right\} .
\end{aligned}
$$

The trace $T^{00}-2 T^{+-}$vanishes, and Eq. (34) is deviatoric. Referring to the third of Eq. (18), the deviatoric part of the moment tensor is related to one of the spheroidal combinations with $l=2$

$$
S_{2}^{(1) m}=\tau_{2}^{0 m}+\frac{1}{2} 3^{1 / 2}\left(\tau_{2}^{+m}+\tau_{2}^{-m}\right)
$$

Following a similar procedure, we can calculate the $L$-th traction tensors

$$
T^{\alpha \beta_{1} \cdots \beta_{L}}=-\int t^{\alpha} x^{\beta_{1}} \cdots x^{\beta_{L}} \mathrm{~d} S
$$

which are $(L+1)$-th order tensors. It is sufficient to note that we have linear combinations

$$
\sum_{\gamma}(-1)^{\gamma}\left\langle\begin{array}{ccc}
l & 1 & L \\
-\gamma & \gamma & 0
\end{array}\right) \tau_{l}^{\gamma m} .
$$

For $L=1$, Eq. (37) reduces to $\gamma$ dependence of Eq. (28).

Due to the selection rules, $l$ takes $L+1, L$ and $L-1$. Except constant factors, Eq. (37) reduces to

$$
S_{L+1}^{(1) m}, \quad T_{L}^{m} \text { and } S_{L-1}^{(2) m}
$$

where 


$$
\begin{aligned}
& S_{l}^{(1) m}=\tau_{l}^{0 m}+[(l+1) / 2 l]^{1 / 2}\left(\tau_{l}^{+m}+\tau_{l}^{-m}\right) \\
& S_{l}^{(2) m}=\tau_{l}^{0 m}-[l / 2(l+1)]^{1 / 2}\left(\tau_{l}^{+m}+\tau_{l}^{-m}\right) \\
& T_{l}^{m}=\tau_{l}^{+m}-\tau_{l}^{-m} .
\end{aligned}
$$

In summary, the single force is related to $S_{1}^{(1) m}$, and the moment tensor is related to $S_{0}^{(2) 0}, T_{1}^{m}$ and $S_{2}^{(1) m}$. Conversely, if the traction is specified at the source radius, an equivalent single force and moment tensor is readily obtained and we can compute seismic displacement.

We note finally that the results of this section may be obtained alternatively in terms of the vector spherical harmonics (Edmonds, 1960).

\section{REFERENCES}

Abramowitz, M. and I. A. Stegun, Handbook of Mathematical Functions, Dover, New York, 1965. Aki, K. and P. G. Richards, Quantitative Seismology, Theory and Methods, W. H. Freeman, San Francisco, 1980.

Backus, G. and M. Mulcahy, Moment tensors and other phenomenological descriptions of seismic sources-I. continuous displacements, Geophys. J. R. Astron. Soc., 46, 341-361, 1976.

Edmonds, A. R., Angular Momentum in Quantum Mechanics, Princeton University Press, New Jersey, 1960.

Dziewonski, A. M., T. A. Chou, and J. H. Woodhouse, Determination of earthquake source parameters from waveform data for studies of global and regional seismicity, J. Geophys. Res., 86, 2825-2852, 1981.

Gilbert, F. and A. M. Dziewonski, An application of normal mode theory to the retrieval of structural parameters and source mechanisms from seismic spectra, Philos. Trans. R. Soc. Lond., A278, 187-269, 1975.

Kanamori, H. and J. W. Given, Analysis of long-period seismic waves excited by the May 18, 1980 eruption of Mt. St. Helens: a terrestrial monopole?, J. Geophys. Res., 87, 5422-5432, 1982.

Kawakatsu, H., Centroid single force inversion of seismic waves generated by landslides, $J$. Geophys. Res., 94, 12363-12374, 1989.

Mochizuki, E., Sphere-resonance method to determine elastic constants of crystal, J. Appl. Phys., 63, 5668-5673, 1988.

Phinney, R. A. and R. Burridge, Representation of the elastic-gravitational excitation of a spherical Earth model by generalized spherical harmonics, Geophys. J. R. Astron. Soc., 34, 451-487, 1973.

Takei, Y., Statistical mechanics of solid-melt composite and the earthquake occurrence by release of gravitational energy, Master Thesis, the University of Tokyo, 1991.

\section{APPENDIX A}

It is shown in this appendix that the first of Eq. (16) and the second of Eq. (18) are valid even for an anisotropic medium.

Vol. 43, No. 1, 1995 
In the vicinity of the source $(r \sim 0)$, we can use the static solution $(\omega \sim 0)$. We first consider the single force

$$
\sigma_{i j, j}+f_{i} \delta(x)=0,
$$

where $\sigma_{i j}$ is the stress tensor, and $\delta$ is the Dirac delta function. If we integrate Eq. (A1) in terms of the Grauss theorem, we obtain the first of Eq. (16) by using $t_{i}=\sigma_{i j} n_{j}$ and Eq. (12).

For the moment tensor, the static equation is given by

$$
\sigma_{i j, j}-\left[M_{i j} \delta(x)\right]_{, j}=0 .
$$

We integrate Eq. (A2) multiplied by $x_{k}$ to obtain

$$
T_{i j}=M_{i j}-\int \sigma_{i j} \mathrm{~d} V
$$

The volume integral is irrelevant for the anti-symmetric part, and we obtain the second of Eq. (18).

We rewrite Eq. (A3) as

$$
T_{i j}=M_{i j}-\int C_{i j k l} u_{k} n_{l} \mathrm{~d} S
$$

where $C_{i j k l}$ is the elastic tensor. For the isotropic case, the surface integral is readily evaluated, and we obtain the first and third of Eq. (18).

\section{APPENDIX B}

\section{Normal-Mode Excitation}

In this appendix, explicit formulae are given for the excitation of seismic normal modes by the moment tensor which is not necessarily symmetric. Formulae for the single force are given in Kawakatsu (1989).

The excitation in terms of the moment tensor $M_{i j}$ is proportional to (e.g., Backus and Mulcahy, 1976).

$$
M_{i j} u_{i, j}^{*}
$$

where $u_{i}$ is the displacement of normal modes, the asterisk denotes complex conjugation, and the summation convention is assumed for repeated indices. The spatial derivative $u_{i, j}$ is evaluated at the source. To compare with well-known results for the symmetric moment tensor, we define the symmetric and anti-symmetric parts with different notation from Eq. (7)

$$
\begin{aligned}
& S_{i j}=\frac{1}{2}\left(M_{i j}+M_{j i}\right) \\
& A_{i j}=\frac{1}{2}\left(M_{i j}-M_{j i}\right) .
\end{aligned}
$$


Also we use the strain and the rotation

$$
\begin{aligned}
& \varepsilon_{i j}=\frac{1}{2}\left(u_{i, j}+u_{j, i}\right) \\
& \omega_{i j}=\frac{1}{2}\left(u_{i, j}-u_{j, i}\right) .
\end{aligned}
$$

Using Eqs. (B2) and (B3), we rewrite Eq. (B1) as

$$
S_{i j} \varepsilon_{i j}^{*}+A_{i j} \omega_{i j}^{*}
$$

where the strain and the rotation are evaluated at the source. The first term due to the symmetric moment tensor has been studied (Phinney and Burridge, 1973; Gilbert and Dziewonski, 1975). The second term is discussed in this appendix.

Seismic normal modes of a spherically symmetric Earth are divided into spheroidal and toroidal modes. The displacement of spheroidal modes is given by

$$
\begin{aligned}
& u_{r}=U Y_{l}^{m} \\
& u_{\theta}=V \partial_{\theta} Y_{l}^{m} \\
& u_{\phi}=V(\sin \theta)^{-1} \partial_{\phi} Y_{l}^{m},
\end{aligned}
$$

where $(r, \theta, \phi)$ are spherical coordinates, $U(r)$ and $V(r)$ are radial eigenfunctions, $Y_{l}^{m}$ are spherical harmonics with angular order $l$ and azimuthal order $m$, and $\partial_{\theta}$ denotes the derivative with respect to $\theta$. The displacement of toroidal modes is

$$
\begin{aligned}
& u_{r}=0 \\
& u_{\theta}=W(\sin \theta)^{-1} \partial_{\phi} Y_{l}^{m} \\
& u_{\phi}=-W \partial_{\theta} Y_{l}^{m},
\end{aligned}
$$

where $W(r)$ are radial eigenfunctions.

To calculate the spatial derivative of the displacement, we use the formalism of Phinney and Burridge (1973). We define the canonical components

$$
\begin{aligned}
& u^{0}=u_{r} \\
& u^{ \pm}=2^{-1 / 2}\left(\mp u_{\theta}+i u_{\phi}\right) .
\end{aligned}
$$

Substituting Eqs. (B5) and (B6) into Eq. (B7), we obtain for spheroidal modes

$$
\begin{aligned}
& u^{0}=\gamma_{l} U Y_{l}^{0 m} \\
& u^{ \pm}=\gamma_{l} \Omega_{1} V Y_{l}^{ \pm 1 m}
\end{aligned}
$$

and for toroidal modes

$$
\begin{aligned}
& u^{0}=0 \\
& u^{ \pm}= \pm i \gamma_{l} \Omega_{1} W Y_{l}^{ \pm 1 m} .
\end{aligned}
$$

Here $\gamma_{l}=[(2 l+1) / 4 \pi]^{1 / 2}$ and $\Omega_{N}=[(l+N)(l-N+1) / 2]^{1 / 2}$. The canonical components $u^{N}$ are expressed in terms of the generalized spherical harmonics $Y_{l}^{N m}$ of Phinney and Burridge (1973). In the following, we use alternative functions which are fully normalized 


$$
\hat{Y}_{l}^{N m}=\gamma_{l} Y_{l}^{N m} .
$$

Equations (B8) and (B9) are rewritten as

$$
u^{0}=U \hat{Y}_{l}^{0 m}, \quad u^{ \pm}=\Omega_{1} V \hat{Y}_{l}^{ \pm 1 m}
$$

and

$$
u^{0}=0, \quad u^{ \pm}= \pm i \Omega_{1} W \hat{Y}_{l}^{ \pm 1 m} .
$$

The canonical components of the second-order tensor are defined by the similar way to Eq. (B7). We introduce a matrix $C_{a i}^{+}$to rewrite Eq. (B7) as

$$
u^{\alpha}=C_{\alpha i}^{+} u_{i} \text {. }
$$

For the moment tensor, we have

$$
M^{\alpha \beta}=C_{\alpha i}^{+} C_{\beta j}^{+} M_{i j}
$$

and explicitly

$$
\begin{aligned}
& M^{00}=M_{r r} \\
& M^{ \pm \mp}=-\frac{1}{2}\left(M_{\theta \theta}+M_{\phi \phi}\right) \mp \frac{i}{2}\left(M_{\theta \phi}-M_{\phi \theta}\right) \\
& M^{ \pm 0}=2^{-1 / 2}\left(\mp M_{\theta r}+i M_{\phi r}\right) \\
& M^{0 \pm}=2^{-1 / 2}\left(\mp M_{r \theta}+i M_{r \phi}\right) \\
& M^{ \pm \pm}=\frac{1}{2}\left(M_{\theta \theta}-M_{\phi \phi}\right) \mp \frac{i}{2}\left(M_{\theta \phi}+M_{\phi \theta}\right) .
\end{aligned}
$$

For the symmetric and anti-symmetric parts defined in Eq. (B2), we have respectively

$$
\begin{aligned}
& S^{00}=S_{r r} \\
& S^{+-}=-\frac{1}{2}\left(S_{\theta \theta}+S_{\phi \phi}\right) \\
& S^{ \pm 0}=2^{-1 / 2}\left(\mp S_{\theta r}+i S_{\phi r}\right) \\
& S^{ \pm \pm}=\frac{1}{2}\left(S_{\theta \theta}-S_{\phi \phi}\right) \mp i S_{\theta \phi}
\end{aligned}
$$

and

$$
\begin{aligned}
& A^{+-}=-i A_{\theta \phi} \\
& A^{ \pm 0}=2^{-1 / 2}\left(\mp A_{\theta r}+i A_{\phi r}\right) .
\end{aligned}
$$

The strain and the rotation can be calculated by the formalism of Phinney and Burridge (1973). The canonical components of the strain for spheroidal modes are given by 


$$
\begin{aligned}
& \varepsilon^{00}=\partial_{r} U \hat{Y}_{l}^{0 m} \\
& 2 \varepsilon^{+-}=-F \hat{Y}_{l}^{0 m} \\
& 2 \varepsilon^{ \pm 0}=\Omega_{1} X \hat{Y}_{l}^{ \pm 1 m} \\
& \varepsilon^{ \pm \pm}=\Omega_{1} \Omega_{2} \frac{V}{r} \hat{Y}_{l}^{ \pm 2 m}
\end{aligned}
$$

with

$$
\begin{aligned}
& F=\frac{1}{r}[2 U-l(l+1) V] \\
& X=\partial_{r} V-\frac{V}{r}+\frac{U}{r}
\end{aligned}
$$

and for toroidal modes

$$
\begin{aligned}
& \varepsilon^{00}=\varepsilon^{+-}=0 \\
& 2 \varepsilon^{ \pm 0}= \pm i \Omega_{1} Z \hat{Y}_{l}^{ \pm 1 m} \\
& \varepsilon^{ \pm \pm}= \pm i \Omega_{1} \Omega_{2} \frac{W}{r} \hat{Y}_{l}^{ \pm 2 m}
\end{aligned}
$$

with

$$
Z=\partial_{r} W-\frac{W}{r}
$$

The canonical components of the rotation for spheroidal modes are

$$
\begin{aligned}
& \omega^{+-}=0 \\
& 2 \omega^{ \pm 0}=\Omega_{1} \tilde{X} \hat{Y}_{l}^{ \pm 1 m}
\end{aligned}
$$

with

$$
\tilde{X}=\partial_{r} V+\frac{V}{r}-\frac{U}{r}
$$

and for toroidal modes

$$
\begin{aligned}
& 2 \omega^{+-}=i l(l+1) \frac{W}{r} \hat{Y}_{l}^{0 m} \\
& 2 \omega^{ \pm 0}= \pm i \Omega_{1} \tilde{Z} \hat{Y}_{l}^{ \pm 1 m}
\end{aligned}
$$

with

$$
\tilde{Z}=\partial_{r} W+\frac{W}{r}
$$

We substitute Eqs. (B18) and (B22) into the first and second terms of Eq. (B4) to 
obtain the excitation of spheroidal modes by the symmetric and anti-symmetric parts of the moment tensor, respectively,

$$
\begin{aligned}
S_{i j} \varepsilon_{i j}^{*}= & S^{\alpha \beta} \varepsilon^{\alpha \beta *} \\
= & \left(S^{00} \partial_{r} U-S^{+-} F\right) \hat{Y}_{l}^{0 m *} \\
& +S^{+0} \Omega_{1} X \hat{Y}_{l}^{1 m *}+S^{-0} \Omega_{1} X \hat{Y}_{l}^{-1 m *} \\
& +S^{++} \Omega_{1} \Omega_{2} \frac{V}{r} \hat{Y}_{l}^{2 m *}+S^{--} \Omega_{1} \Omega_{2} \frac{V}{r} \hat{Y}_{l}^{-2 m *} \\
& A^{\alpha \beta} \omega^{\alpha \beta *}=A^{+0} \Omega_{1} \tilde{X} \hat{Y}_{l}^{1 m *}+A^{-0} \Omega_{1} \tilde{X} \hat{Y}_{l}^{-1 m *} .
\end{aligned}
$$

Similarly we obtain the excitation of toroidal modes

$$
\begin{gathered}
S^{\alpha \beta} \varepsilon^{\alpha \beta *}=i\left(-S^{+0} \Omega_{1} Z \hat{Y}_{l}^{1 m *}+S^{-0} \Omega_{1} Z \hat{Y}_{l}^{-1 m *}\right. \\
\left.-S^{++} \Omega_{1} \Omega_{2} \frac{W}{r} \hat{Y}_{l}^{2 m *}+S^{--} \Omega_{1} \Omega_{2} \frac{W}{r} \hat{Y}_{l}^{-2 m *}\right) \\
A^{\alpha \beta} \omega^{\alpha \beta *}=i\left[-A^{+-} l(l+1) \frac{W}{r} \hat{Y}_{l}^{0 m *}\right. \\
\left.-A^{+0} \Omega_{1} \tilde{Z} \hat{Y}_{l}^{1 m *}+A^{-0} \Omega_{1} \tilde{Z} \hat{Y}_{l}^{-1 m *}\right] .
\end{gathered}
$$

In Eqs. (B26)-(B29), radial eigenfunctions are evaluated at the source depth.

Derivatives of strain and rotation are necessary in the centroid-moment tensor inversion (Dziewonski et al., 1981). For spheroidal modes, derivatives are given by

$$
\begin{aligned}
& \varepsilon^{00,0}=\partial_{r}^{2} U \hat{Y}_{l}^{0 m} \\
& 2 \varepsilon^{+-, 0}=-\partial_{r} F \hat{Y}_{l}^{0 m} \\
& 2 \varepsilon^{ \pm 0,0}=\Omega_{1} \partial_{r} X \hat{Y}_{l}^{ \pm 1 m} \\
& \varepsilon^{ \pm \pm, 0}=\Omega_{1} \Omega_{2} \frac{1}{r}\left(\partial_{r} V-\frac{V}{r}\right) \hat{Y}_{l}^{ \pm 2 m} \\
& \varepsilon^{00, \pm}=\Omega_{1} \frac{1}{r}\left[\left(\partial_{r} U-\frac{U}{r}\right)-\left(\partial_{r} V-\frac{V}{r}\right)\right] \hat{Y}_{l}^{ \pm 1 m} \\
& 2 \varepsilon^{+-, \pm}=-\Omega_{1} \frac{1}{r}(X+F) \hat{Y}_{l}^{ \pm 1 m} \\
& 2 \varepsilon^{ \pm 0, \mp}=\frac{1}{r}\left[\frac{1}{2} l(I+1)\left(\partial_{r} V-\frac{3}{r} V+\frac{U}{r}\right)-2\left(\partial_{r} U-\frac{U}{r}\right)\right] \hat{Y}_{l}^{0 m} \\
& 2 \varepsilon^{ \pm 0, \pm}=\Omega_{1} \Omega_{2} \frac{1}{r}\left(\partial_{r} V-\frac{3}{r} V+\frac{U}{r}\right) \hat{Y}_{l}^{ \pm 2 m}
\end{aligned}
$$




$$
\begin{aligned}
& \varepsilon^{ \pm \pm, \mp}=-\Omega_{1} \frac{1}{r}\left(\partial_{r} V+\frac{1}{2} F\right) \hat{Y}_{l}^{ \pm 1 m} \\
& \varepsilon^{ \pm \pm, \pm}=\Omega_{1} \Omega_{2} \Omega_{3} r^{-2} V \hat{Y}_{l}^{ \pm 3 m}
\end{aligned}
$$

and

$$
\begin{aligned}
& 2 \omega^{+-, 0}=0 \\
& 2 \omega^{ \pm 0,0}=\Omega_{1} \partial_{r} \tilde{X} \hat{Y}_{l}^{ \pm 1 m} \\
& 2 \omega^{+-, \pm}=\mp \Omega_{1} r^{-1} \tilde{X} \hat{Y}_{l}^{ \pm 1 m} \\
& 2 \omega^{ \pm 0, \mp}=\frac{1}{2} l(l+1) r^{-1} \tilde{X} \hat{Y}_{l}^{0 m} \\
& 2 \omega^{ \pm 0, \pm}=\Omega_{1} \Omega_{2} r^{-1} \tilde{X} \hat{Y}_{l}^{ \pm 2 m}
\end{aligned}
$$

For toroidal modes, the derivatives are

$$
\begin{aligned}
& \varepsilon^{00,0}=\varepsilon^{+-, 0}=0 \\
& 2 \varepsilon^{ \pm 0,0}= \pm i \Omega_{1} \partial_{r} Z \hat{Y}_{l}^{ \pm 1 m} \\
& \varepsilon^{ \pm \pm, 0}= \pm i \Omega_{1} \Omega_{2} \frac{Z}{r} \hat{Y}_{l}^{ \pm 2 m} \\
& \varepsilon^{00, \pm}=\mp i \Omega_{1} \frac{Z}{r} \hat{Y}_{l}^{ \pm 1 m} \\
& 2 \varepsilon^{+-, \pm}=\mp i \Omega_{1} \frac{Z}{r} \hat{Y}_{l}^{ \pm 1 m} \\
& 2 \varepsilon^{ \pm 0, \mp}= \pm i \frac{1}{2} l(l+1) \frac{Z}{r} \hat{Y}_{l}^{0 m} \\
& 2 \varepsilon^{ \pm 0, \pm}= \pm i \Omega_{1} \Omega_{2} \frac{1}{r}\left(\partial_{r} W-\frac{3}{r} W\right) \hat{Y}_{l}^{ \pm 2 m} \\
& \varepsilon^{ \pm \pm, \mp}=\mp i \Omega_{1} \frac{1}{r}\left[\partial_{r} W-\frac{1}{2} l(l+1) \frac{W}{r}\right] \hat{Y}_{l}^{ \pm 1 m} \\
& \varepsilon^{ \pm \pm, \pm}= \pm i \Omega_{1} \Omega_{2} \Omega_{3} r^{-2} W \hat{Y}_{l}^{ \pm 3 m}
\end{aligned}
$$

and

$$
\begin{aligned}
& 2 \omega^{+-, 0}=i l(l+1) \frac{Z}{r} \hat{Y}_{l}^{0 m} \\
& 2 \omega^{ \pm 0,0}= \pm i \Omega_{1} \partial_{r} \tilde{Z} \hat{Y}_{l}^{ \pm 1 m} \\
& 2 \omega^{+-, \pm}=-i \Omega_{1} \frac{1}{r}\left[\partial_{r} W-l(l+1) \frac{W}{r}+\frac{W}{r}\right] \hat{Y}_{l}^{ \pm 1 m} \\
& 2 \omega^{ \pm 0, \mp}= \pm i \frac{1}{2} l(l+1) \frac{Z}{r} \hat{Y}_{l}^{0 m} \\
& 2 \omega^{ \pm 0, \pm}= \pm i \Omega_{1} \Omega_{2} r^{-1} \tilde{Z} \hat{Y}_{l}^{ \pm 2 m} .
\end{aligned}
$$

Vol. 43, No. 1, 1995 